\title{
PET Evaluation of Microglial Activation in Non-neurodegenerative Brain Diseases
}

\author{
Christine Ghadery ${ }^{1} \cdot$ Laura A. Best $^{2} \cdot$ Nicola Pavese $^{2,3} \cdot$ Yen Foung Tai ${ }^{4} \cdot$ Antonio P. Strafella $^{1}$
}

Published online: 28 May 2019

(C) The Author(s) 2019

\begin{abstract}
Purpose of the Review Microglial cell activation is an important component of neuroinflammation, and it is generally well accepted that chronic microglial activation is indicative of accumulating tissue damage in neurodegenerative conditions, particularly in the earlier stages of disease. Until recently, there has been less focus on the role of neuroinflammation in other forms of neurological and neuropsychiatric conditions. Through this review, we hope to demonstrate the important role TSPO PET imaging has played in illuminating the pivotal role of neuroinflammation and microglial activation underpinning these conditions.

Recent Findings TSPO is an $18 \mathrm{kDa}$ protein found on the outer membrane of mitochondria and can act as a marker of microglial activation using nuclear imaging. Through the development of radiopharmaceuticals targeting TSPO, researchers have been able to better characterise the spatial-temporal evolution of chronic neurological conditions, ranging from the focal autoimmune reactions seen in multiple sclerosis to the Wallerian degeneration at remote parts of the brain months following acute cerebral infarction.

Summary Development of novel techniques to investigate neuroinflammation within the central nervous system, for the purposes of diagnosis and therapeutics, has flourished over the past few decades. TSPO has proven itself a robust and sensitive biomarker of microglial activation and neuroimaging affords a minimally invasive technique to characterise neuroinflammatory processes in vivo.
\end{abstract}

Keywords Neuroinflammation $\cdot$ Microglial activation $\cdot$ TSPO $\cdot$ PET $\cdot$ Neurological diseases $\cdot$ Neuropsychiatric diseases

Christine Ghadery and Laura A. Best contributed equally to this work.

This article is part of the Topical Collection on Neuroimaging

Laura A. Best

Laura.best@newcastle.ac.uk

1 The Edmond J. Safra Program in Parkinson's Disease \& Movement Disorder Unit, Toronto Western Hospital \& Krembil Research Institute, University Health Network; Research Imaging Centre, Campbell Family Mental Health Research Institute, Centre for Addiction and Mental Health, University of Toronto, Toronto, Ontario, Canada

2 Clinical Ageing Research Unit, Newcastle University, Campus for Ageing and Vitality, Westgate Road, Newcastle Upon Tyne, UK

3 PET centre, University of Aarhus Denmark, Aarhus, Denmark

4 Imperial College London South Kensington Campus, London, UK

\section{Introduction}

Microglia constitute approximately $5-10 \%$ of adult brain cells and are found throughout brain tissue $[1 \bullet \bullet$. They are the resident immune cells of the brain and represent an important effector in the innate immunity of the brain $[1 \bullet \cdot$. Their capacities, including phagocytosis, proliferation and secretion of soluble molecules, mean they are implicated in the neuroinflammatory pathological processes underpinning a number of neurological and neuropsychiatric conditions.

Microglia become "activated" when the integrity of the central nervous system (CNS) is challenged, for example, in the presence of vascular or tissue damage $[2 \bullet \cdot$. Under these circumstances, microglia shift from a sensing state to a reactive state, triggering the release of cytokines, proteinases, complement proteins and reactive oxygen species [3]. The reactive phenotype can then be further subdivided into a pro-inflammatory state (M1) or one characterised by antiinflammatory reactions (M2) supporting tissue repair, 
regeneration and coordination of the immune response $[2 \bullet \bullet]$. Polarisation of microglial cells is a dynamic, context-specific process depending on the type of stimulus, with temporal integration determining the neuroinflammatory changes seen in the acute versus the chronic setting $[4,5]$.

Targeted positron emission tomography (PET) imaging of microglia facilitates the in vivo assessment of neuroinflammation through the development of radiopharmaceuticals that target biomarkers of microglial activation. Paramount amongst these is the translocator protein $18-\mathrm{kDa}$ (TSPO), which was previously described as a peripheral benzodiazepine receptor following its identification in through binding studies using ${ }^{3} \mathrm{H}$-diazepam [6]. Under normal circumstances, TSPO is moderately expressed in healthy brain tissue and only minimally expressed in resting microglia. However, with the homeostatic disturbance that inevitably accompanies neuroinflammation, TSPO expression substantially increases, and this is predominantly observed in activated microglia [7]. TSPO upregulation has also been observed in astrocytes, for example following cerebral ischaemia [8], and macrophages that infiltrate the damaged brain due to disruption of the blood-brain barrier and increased vascular permeability secondary to neuroinflammation [9].

The exact role of TSPO, however, remains unclear. It is postulated to be involved in a number of cellular functions ranging from regulation of cell proliferation to cell apoptosis, and its function in the stimulation of microglia is also poorly understood, with most studies suggesting a possible role in reactive oxygen species attenuation $[2 \cdot \bullet, 10,11]$. Despite the lack of functional clarity, TSPO upregulation remains a relevant biomarker, and longitudinal studies utilising TSPO PET have provided insights into the temporal dynamics of neuroinflammation leading to neuronal injury and the progression of chronic disease. Furthermore, TSPO PET allows researchers to determine the efficacy of emerging antiinflammatory therapies.

Over the past two decades a number of TSPO ligands have emerged, which can be subdivided into first, second and third generation ligands. The most prominent, and still most widely used, is the first generation TSPO ligand ${ }^{11} \mathrm{C}-\mathrm{PK} 11195$ (PK). PK shows high affinity for binding to TSPO; however, its clinical utility is somewhat limited by the relatively short half-life of carbon-11, requiring an on-site cyclotron, poor signal-to-noise ratio as a consequence of high non-specific binding and low brain bioavailability $[12,13 \bullet]$.

In an attempt to overcome the difficulties with PK in the pursuit of improved image quality, investigators sought to develop superior ligands, including phenoxyarylactemides derivatives (e.g. ${ }^{11} \mathrm{C}-\mathrm{PBR} 28,{ }^{11} \mathrm{C}$-DAA1106 and ${ }^{18} \mathrm{~F}$-PBR06), pyrazolopyrimidines derivatives (e.g. ${ }^{18} \mathrm{~F}$-DPA-714) and imidazopyridine derivatives (e.g. ${ }^{11}$ C-CLINME) [2••]. However, the second-generation ligands have not been without their own drawbacks, and the most significant concerns their sensitivity to the rs6971 polymorphism (Ala147Thr) in the TSPO gene. This affects binding affinity properties causing significant heterogeneity in PET imaging and skewing interpretation of the associated quantitative data. Consequentially, the third-generation radioligands have emerged in an attempt to develop rs6971-insensitive ligands. These TSPO tracers include flutriciclamide $\left({ }^{18} \mathrm{~F}\right.$-GE180) [14*. ] and ${ }^{11}$ C-ER176 [15]; however, their clinical relevance is still to be determined.

In this review, we will discuss how TSPO PET imaging has been utilised to characterise the neuroinflammatory processes key to the pathogenesis to a number of varied neurological and neuropsychiatric conditions. We will combine the data from studies using PK and newer generation TSPO ligands to provide a detailed overview of how this research has led to a better understanding of the temporal and spatial relationships in microglial activation, neuroinflammation and disease manifestation. We will also discuss some of the limitations in TSPO PET imaging and suggestions for future progress in this field leading to a more personalised approach to treatment of neuroinflammation.

\section{Multiple Sclerosis}

Multiple sclerosis (MS) is an autoimmune chronic condition characterised by migration of myelin-reactive T-cells, with subsequent microglial and astrocyte activation and recruitment of peripherally circulating macrophages causing demyelination and oligodendrocyte destruction within the CNS [16]. While MRI demonstration of lesions disseminated in time and space remains the diagnostic gold standard, TSPO PET imaging is a potentially promising tool not only in the diagnosis of MS, but in detecting the conversion from relapsing-remitting MS (RRMS) to secondary progressive MS (SPMS).

Studies have shown that activated microglia play a central role in sustaining chronic neuroinflammation in MS [17] and are found to have a specific and more diffuse distribution in SPMS when compared with RRMS [18, 19•]. In SPMS, greater mean TSPO radioligand uptake has been demonstrated throughout the white matter, as well as, specifically in the deep grey matter and the thalami of patients with SPMS [18, 19•, 20]. This increased cortical binding appears to correlate with disability, including impaired cognitive performance [18, 19•]. Further studies suggest that there is a positive correlation between TSPO ligand binding and disease severity [21, 22] and duration [20,23]; however, the results are not consistent. In active disease, increased PK binding has been found to correspond to MRI gadolinium-defined active lesions, but not in chronic lesions $[24,25]$.This corresponds to similar findings by $\mathrm{Oh}$ and colleagues using the ${ }^{11} \mathrm{C}$-PBR28 ligand, which demonstrated that gadolinium-enhancing lesions had significantly higher binding compared with the contralateral 
white matter [23]. Additionally, PK binding in seemingly normal white matter has not only been found to have increased density in RRMS and SPMS patients compared with healthy controls, but it also appears to correlate with the degree of observed cerebral atrophy [26].

In clinically isolated syndrome (CIS), PK binding has been shown to have a potentially prognostic role. Gianetti and colleagues demonstrated that in patients with CIS who went on to develop MS within 2 years had higher PK binding on their baseline scans [22]. It has also been demonstrated that TSPO PET can be utilised to predict the development of MRI active lesions, suggesting microglial activation may play a pivotal role in MS lesion formation [23].

In general, PET imaging using second-generation TSPO tracers has been less successful in yielding results useful to the understanding of MS [27-29]. However, not all of the studies considered the implications of the rs6971 polymorphism in their analysis [28]. When this is taken into consideration, a study using the ${ }^{18} \mathrm{~F}$-PBR 111 radioligand revealed increased total distribution volume $\left(\mathrm{V}_{\mathrm{T}}\right.$, i.e. the ratio of radioligand concentration in the tissue target region to that in blood plasma at equilibrium) in MS patients compared with controls. There results also suggested an association between TSPO PET white matter signal and disease severity [21]. Similarly, Singhal and colleagues using the ${ }^{18}$ F-PBR06 ligand demonstrated its utility in assessing TSBO binding in MS [30, 31•]. They showed a significant correlation between microglial activation in deep grey matter, cerebellar white matter and white matter lesions associated with neurological disability and cerebral atrophy.

Finally, animal models have demonstrated that following treatment with immunosuppressive drugs, such as fingolimod, TSPO radiotracer uptake is reduced [32]. Similarly, clinical studies on patients with MS, published by Ratchford and colleagues, demonstrated that PK binding potential per unit volume was significantly decreased throughout the brain following treatment with glatiramer acetate after 1 year [33]. Potentially, therefore, TSPO PET could be used as a noninvasive biomarker to determine and monitor the efficacy of immunosuppressive therapies on MS disease activity.

\section{Stroke}

Stroke researchers have utilised TSPO PET imaging to understand the role and time course of neuroinflammation following acute cerebral infarction. We know that following acute cerebral hypoxia, there is a significant increase in TSPO expression, most notably in astrocytes and microglia [34], with the inflammatory response divided into an initial release of proinflammatory mediators followed by a later neuroprotective phase distinguished by the release of anti-inflammatory mediators [35]. However, contradictory results [36] to this model suggest a much more complex and heterogeneously dynamic process making the development of immunomodulatory therapies problematic. Further complicating this, is the observation that neuroinflammation is not localised to the immediate surroundings of the infarct, but is also observed in remote brain regions that have fibre tract connections with the acutely affected area [37]. Activated microglia around areas of acute infarction exhibit distinct immunohistochemical properties compared with microglia distant from the lesion in the chronic stages following stroke [38]. Studies have demonstrated that areas remote to the infarcted area show evidence of extensive phagocytosis and iron deposition, compared with the relative resolution of neuroinflammation at the lesional site several months post-event [39, 40]. Such observations emphasise the need for reliable imaging biomarkers targeting neuroinflammation.

Studies using PK PET complement the immunohistochemical findings for the acute and chronic stages of stroke. Within a few days of the insult, increased PK binding is observed, with activated microglia present in the peri-infarct zone progressing to the ischemic zone a few days later [41]. The activation typically peaks approximately 1 week following the stroke, and thereafter it decreases in the acute region [42]. However, as with the immunohistochemistry, repetitive PK studies demonstrate marked neurodegeneration at sites remote from the lesion site as time post-event elapses. Evidence for this comes from a number of studies [37, 39, 40, 43] and is felt to represent Wallerian degeneration along connected pathways between different anatomical areas [44]. Thiel and colleagues described the temporal dynamics of locally activated microglia and their relationship to pyramidal tract damage in patients with subcortical stroke. They found that microglial activity at the infarct site decreases with recovery, with uptake ratios not significantly different to controls after 6 months, while activity persists in the brainstem along the affected pyramidal tract [37]. Similarly, Walberer and colleagues investigated neuroinflammation in the chronic stage of embolic stroke using a rat model and the PK ligand. The authors demonstrated that neuroinflammation all but resolved at the lesion site at 7 months, but with microglia activation detected at sites remote from the primarily infarcted regions, including the ipsilateral thalamus [39].

A potential limitation with the PK ligand regards its ability to differentiate between the infract and the peri-infarct region, in part due to low signal-to-noise ratio and non-specific binding. A number of comparative studies have aimed to improve the diagnostic power of TSPO PET using newer ligands, with differing degrees of success. Guylas and colleagues using ${ }^{11} \mathrm{C}$ vinpocentine measured regional changes of TSPO in the brain of nine ischemic stroke patients at nine different points between 1 and 14 weeks after the event [41]. At 1 week poststroke, they observed an increased ${ }^{11} \mathrm{C}$-vinpocentine uptake in both the ischemic core and the peri-infract zone, with microglial activation seemingly more intense in the peri- 
infarct area. This increased uptake then decreased steadily with post-stroke time. Unfortunately, however, the authors noted that ${ }^{11} \mathrm{C}$-vinpocentine demonstrated low affinity for TSPO and in a further study, directly comparing ${ }^{11} \mathrm{C}$ vinpocentine with $\mathrm{PK}$, they found that although ${ }^{11} \mathrm{C}$ vinpocentine had a greater affinity for TSPO than PK, the differences were not significant [45].

More promise has been seen with the ${ }^{18}$ F-DPA-714 and ${ }^{11} \mathrm{C}$-DPA-713 ligands. In one such study, nine patients underwent PET imaging using the ${ }^{18} \mathrm{~F}$-DPA-714 radio-ligand between 8 and 18 days after stroke [46]. The authors demonstrated that observed increases in ligand uptake co-localised with the infarcted tissue and extension beyond the region corresponding to the damage in the blood-brain barrier. This suggests that ${ }^{18} \mathrm{~F}$-DPA-714 may be useful in assessing the extent of neuroinflammation associated with acute stroke, although it must be noted that no correlation was identified between tracer uptake and infarct volume. A recently published study by Chaney and colleagues, where mice were subject to middle cerebral artery occlusion or sham surgery, compared the two second-generation ligands ${ }^{11} \mathrm{C}$-DPA-713 and ${ }^{18} \mathrm{~F}-\mathrm{GE}-180$ [47•]. While both ligands were able to detect neuroinflammation at acute and chronic time points following the ischaemic event, ${ }^{11} \mathrm{C}$-DPA-713 was found to be more sensitive in reflecting the extent of glial cell activation and allowed earlier detection compared with ${ }^{18}$ F-GE-180 [47•]. Such findings support TSPO as a useful biomarker of neuroinflammation in distinguishing the sub-acute and chronic phases poststroke.

Both PK and newer TSPO ligands have been key in elucidating how changes in TSPO expression, both spatially and temporally, relate to the pathogenesis of stroke and highlight a conceivable role for novel anti-inflammatory substances in the long-term management of stroke. Furthermore, TSPO ligands, such as ${ }^{18} \mathrm{~F}$-DPA-714 and ${ }^{11} \mathrm{C}$-DPA-713, have a potential role in tracking in vivo microglial activation, which may allow predictions regarding individual functional recovery and assessing the utility of future therapeutic strategies $[46,48]$.

\section{Traumatic Brain Injury}

Traumatic brain injury (TBI) is a disease with many different symptoms including cognitive, emotional and physical impairments. In general, the majority of TBIs are single events; however, repeated injuries among affected people are related to the development of chronic traumatic encephalopathy (CTE), even though the pathology of CTE can also be seen after single TBI [49॰]. Mechanisms responsible for these disabilities can be divided into primary and secondary injuries. While the primary injury is the initial biomechanical trauma [50], a process involving neuronal, axonal and vascular damage induced by the kinetic energy, this induces a cascade of secondary processes leading to excitotoxicity, necrosis, apoptosis, autophagy and free radical formation [51]. Hence, TBI is considered to be a chronic disease [52], implicating that the pathophysiological and inflammatory processes in the brain take place at different times after the injury, where some are beneficial to recovery and others are generated by the injury and exaggerate the primary damage $[53,54]$.

Brain injury can trigger neurodegeneration and is considered a major riskfactor for the development of dementia, as highlighted in one study $\mathrm{b} y$ the accumulation of amyloid- $\beta$ plaques in around $30 \%$ of post-mortem brain tissue collected from TBI patients [55]. Interestingly, PET imaging studies in TBI patients revealed a similar distribution of amyloid plaques as in patients with Alzheimer's disease [56]. Further, TBI has been associated with Parkinson's disease [57] and various psychiatric disorders including increased risk of suicide, and overall increase in mortality [58].

Areas of activated microglia often coincide with observed neuronal degeneration and axonal abnormality [59,60]. Studies in animal models [61] and in humans [62,63] have detected microglial activation occurring early after TBI, but then persisting for years, detectable both in vivo and postmortem. A study by Ramlackhansingh and colleagues using PK in patients 11 months to 17 years post-TBI found binding to be significantly increased in the thalami, occipital cortices, putamen and posterior limb of the internal capsules, but with no increase at the original site of injury [62]. High PK binding in the thalamus corresponded to worse cognitive outcomes and increased microglial activation was identified up to 17 years following head injury, supporting the premise that TBI triggers a chronic neuroinflammatory response [62].

These findings have been replicated in further studies, with one group showing that PK binding was again increased in subcortical regions remote from acute injury site [64] and in models of experimental TBI [53]. The prominence of microglial activation in the subcortical structures may reflect their dense connectivity and suggests that microglia behave differently locally to site of injury from those at remotely connected structures. It is likely that this reflects a slowly progressive process within damaged white matter similar to what is observed in stroke [49•].

Recent investigations using ${ }^{11} \mathrm{C}-\mathrm{DPA}-713$, a secondgeneration TSPO tracer, in older former National Football League (NFL) players revealed significantly higher TSPO PET signal in the right amygdala and bilateral supramarginal gyri of the players compared with controls [65]. Further, Coughlin and colleagues [66 ${ }^{\bullet}$ recently reported increased TSPO binding in predominantly medial temporal lobe regions and subtle evidence of white matter damage on diffusion MRI in a group of 14 active or recently retired NFL players with a history of concussions. Previous PK studies in both TBI and stroke have demonstrated that ligand binding correlated with the extent of white matter damage and axonal injury may be 
an important factor in causing persistent microglial activation leading to progressive degeneration $[49 \bullet, 62]$. Animal models of closed TBI using ${ }^{18}$ F-DAP-714 have demonstrated that uptake correlated with trauma severity, metabolic deficits and the degree of microglial activation [67•].

The clinical significance of persistent microglial activation in TBI is unclear, however one can deduce that the balance of activation states/polarisation varies at different points [49•]. The relevance of microglia in the general pathophysiological response to TBI is potentially therapeutically relevant, and there is interest in targeting microglial pathways and immune modulation to prevent neurodegeneration [68-70]. Longitudinal studies are required to clarify the functional significance of microglial activation in remote parts of the brain and determine the reliability of TSPO ligands as markers for severity and progression in TBI $[62,71]$.

\section{Neuroinflammation in Human Immunodeficiency Virus (HIV) Infection}

Microglia activation appears to play a role in the development of cognitive impairment and dementia associated with HIV infection. In a pilot study, five healthy volunteers and 10 HIV-positive patients, with and without HIV-associated dementia (HAD), underwent PET with the PK ligand [72]. As a group, the HIV-positive patients overall showed significantly higher tracer binding than controls in five brain regions, and similar results have been observed using newer TSPO radioligands, such as ${ }^{11} \mathrm{C}-\mathrm{PBR} 28$ [73•].

Patients with HAD did not show any significant difference in PK-binding compared with HIV-positive, non-demented patients. However, while non-demented HIV-positive patients did not show any significantly increased binding compared with controls, HAD patients demonstrated significantly higher PK-binding than controls in five out of eight brain regions, supporting a possible role for microglial activation in HAD [72]. In contrast to this, another PET study using the PK radioligand in $12 \mathrm{HIV}$-infected patients with minor neurocognitive impairment and 5 controls found no increase in PK-binding in the HIV-infected patients compared with controls in any of the investigated brain regions [74]. The authors concluded that PK-binding PET might be insensitive to the degree of macrophage activation in HIV-associated minor neurocognitive impairment or, alternatively macrophage activation is not implicated in this condition. A third alternative, however, is that the ROI approach used in this study is not sensitive enough to detect subtle and localised increases in activated microglia in cortical areas. In fact, using voxel-by-voxel analysis, Garvey and colleagues detected the presence of activated microglia in several focal cortical areas in asymptomatic HIV-infected patients [75]. Additionally, increased PK-binding in the anterior cingulate, corpus callosum and posterior cingulate correlated with poorer executive performance.
TSPO binding using the ${ }^{11} \mathrm{C}-\mathrm{PBR} 28$ ligand found global increases in TSPO expression, with significant regional increases in the occipital lobe, parietal lobe and globus pallidus in patients who were HIV-positive when compared with controls. This same study found that increased TSPO binding in the amygdala, thalamus and hippocampus correlated with poorer global cognitive performance, particularly with verbal and visual memory [73•]. It remains to be established whether detection of activated microglia in HIV-positive patients is a predictor of future neurocognitive decline.

\section{Neuropsychiatric Disease}

TSPO PET-studies provide evidence that impaired regulation of microglia contributes to both neurobehavioural and neuropsychiatric disorders. In schizophrenia, for example, one of the most consistent genetic associations relating to this disorder concerns the major histocompatibility complex, and therefore the innate immune system [13•]. The pathology of schizophrenia has been associated with neuroinflammation, and the relevance of microglial activation can be observed in the inhibitory effects of both typical and atypical anti-psychotics on activated microglia [76]. Two small studies utilising PK have demonstrated increased TSPO signal in the grey matter, hippocampus and temporal cortex of patients with schizophrenia [77, 78]. Unfortunately, later studies using larger cohorts and new TSPO radioligands have been unable to replicate these results in patients with early-stage psychosis or schizophrenia when compared with healthy controls [79-82]. In one such study, while Takano and colleagues demonstrated a positive correlation between positive symptom scores, disease duration and ${ }^{11} \mathrm{C}$-DAA1106 binding, there was no significant difference in total binding in cortical areas when compared with controls [80]. Another recent study detected no difference in ${ }^{18}$ F-FEPPA binding between 19 untreated patients with firstepisode psychosis compared with 20 controls [83].

However, more promising insight arises from a study utilising the ${ }^{11} \mathrm{C}$-PBR28 radio-ligand. The investigators found that in schizophrenic patients the total cortical grey matter volume was significantly lower when compared with healthy controls [84•]. Patients were genotyped for the rs6971 polymorphism. This corresponded to a negative correlation between TSPO signal and the total cortical grey matter volume. While these findings suggest that in schizophrenia microglial activation is related to altered cortical volume, longitudinal studies will be required to determine the exact relationship between microglial activation and cortical grey matter loss, and whether anti-psychotic treatment has any effect on changes in brain volume observed in these patients.

Investigations in depression have also not yielded conclusive results, with one such study concluding that there was no statistical difference between patients with mild to moderate depression and healthy controls using ${ }^{11}$ C-PBR28 PET [85]. 
Table 1 Limitations of TSPO radiotracers

\begin{tabular}{llc}
\hline First generation TSPO [12,99] & Second generation TSPO & Third generation TSPO \\
\hline Short half-life & rs6971 polymorphism & $\begin{array}{c}\text { Challenge of absolute quantification and } \\
\text { kinetic modelling }\end{array}$ \\
Poor signal-to-noise ratio & No differentiation between M1 and M2 & No differentiation between M1 and M2 \\
Non-specific binding & $\begin{array}{l}\text { Slow accumulation of radio metabolites resulting in } \\
\text { inaccurate estimations of TSPO [100,101] } \\
\text { Slow kinetic behavior necessitating longer scanning time [101] }\end{array}$ & Limited data available \\
& S & \\
\hline
\end{tabular}

However, studies are emerging that challenge this in more severely affected patients and demonstrating the effect of treatment on neuroinflammation. Setiawan and colleagues using the ${ }^{18} \mathrm{~F}$-FEPPA ligand found slightly elevated $\mathrm{V}_{\mathrm{T}}$ values in the anterior cingulate cortex of 20 patients with major depressive episode (MDE) compared with 20 controls [86]. This area is implicated in the control of emotional behaviour, and this finding represents the first evidence of significant increase in brain TSPO density in vivo during MDE [86]. This finding has recently been replicated by Richards and colleagues using the ${ }^{11} \mathrm{C}$-PBR28 TSPO radio-ligand in patients with MDE [87•]. They identified a trend towards increased ${ }^{11} \mathrm{C}-\mathrm{PBR} 28$ binding in patients with MDE compared with healthy controls, and post hoc analysis further demonstrated that this abnormality was significant in the unmedicated MDE patients. Similar findings have also been published regarding PK TSPO binding, which have found that binding was significantly elevated in the anterior cingulate and insula in patients with co-existent MDE and suicidal ideation [88].

In further work, Setiawan and colleagues again demonstrated using 18F-FEPPA PET that TSPO $\mathrm{V}_{\mathrm{T}}$ is increased in patients with more advanced MDE who have had longer periods without anti-depressant treatment compared with those who have had shorter periods without medication [89••]. They further found that TSPO binding was between 29 and $33 \%$ greater in the prefrontal cortex, anterior cingulate cortex and insula in patients with longer disease duration [89••]. These results are strongly suggestive of a different illness phase, which has implications for staging MDE, and the authors also found that the yearly increase in microglial activation they observed in untreated patients (14-18\% per decade) disappeared when anti-depressants were initiated [89••]. This is not surprising knowing that serotonin reuptake inhibitors are shown to inhibit microglial activation [90, 91]. These results, therefore, support the theory that major depression is due to neuropathological progression secondary to chronic microglial activation and are consistent with the observed clinical transition from infrequent solitary episodes, with inter-episode recovery, towards more persistent disease [89••].

A further recent study demonstrated that when ${ }^{18}$ F-FEPPA PET results were compared before and after treatment with cognitive behavioural therapy in patients with $\mathrm{MDE} ; V_{\mathrm{T}}$ values were significantly reduced during the treatment period [92]. Interestingly, reduction in $V_{\mathrm{T}}$ values was not significant in the cohort who received supportive psychotherapy. However there was a correlation observed in both treatment groups between reduction in TSPO $V_{\mathrm{T}}$, consistent in the hippocampus and amelioration of depressive symptoms [92]. Interestingly, studies comparing patients with bipolar disorder to healthy controls using PK also found significantly increased tracer binding in the hippocampus $[93,94]$. Thus, highlighting that hippocampal TSPO overexpression appears to be a shared characteristic between distinct psychiatric disorders.

Certainly, there is an accumulating body of evidence associating the pathophysiology of neuropsychiatric conditions with dysregulation of the immune response. However, the heterogeneity within study populations, as well as relatively small sample sizes, makes replication of studies in mental health research more difficult. Considering the PK and ${ }^{11} \mathrm{C}$ PBR28 findings in schizophrenia, the patients recruited had an established diagnosis, and in a similar pattern to MDE, the results may indicate that the aberrant immune response is representative of the pathophysiology in the chronic phase of illness with progressive change occurring at the glial level. This warrants clarification with further studies.

\section{Limitations of TSPO PET Imaging}

There are a number of limitations concerning the utility of TSPO as a PET microglial biomarker. These include low brain density, expression by cells other than microglia, similar expression of activated microglia in with M1 and M2 states and the incidence of the aforementioned genetic polymorphism. Certainly, it is not clear whether increased or decreased TSPO binding reflects a particular microglial phenotype, meaning that TSPO PET does not differentiate between a specific functional role, i.e. neurotoxic (M1) versus neuroprotective (M2) [1••]. It is, therefore, more accurate to state that upregulation of TSPO depicts the broader multicellular neuroinflammatory reaction, rather than simply reflecting microglia activation [95•]. This statement is supported by studies showing that TSPO expression in both microglia and astrocytes appears to be temporally distinct, depending on the stage of disease progression as much as on the disease itself. For 


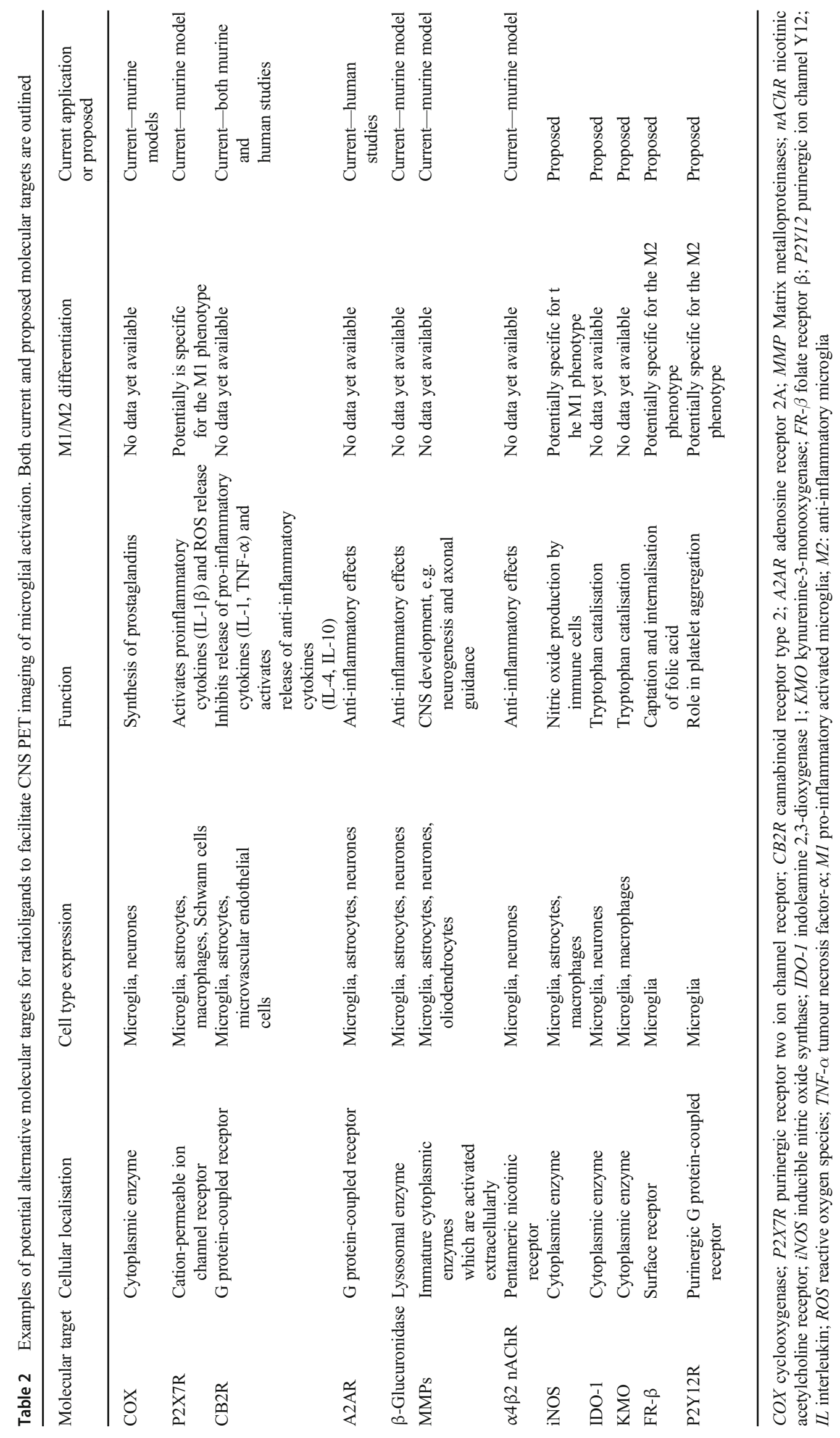


example, in some rat models of active MS, increased TSPO expression is localised to microglial cells [25], in contrast to post-mortem animal and human data demonstrating involvement of both astrocytes and microglia [96, 97]. The presumption is that initial microglial activation is followed by delayed but prolonged astrocytic activation.

Another important consideration is the estimation of specific TSPO binding. Typically, kinetic modelling is the standard for PET quantification; however, this can be challenging with TSPO radioligands $[1 \bullet \cdot$. Studies using both PK and secondgeneration ligands have reported significant between-subject variability in $V_{\mathrm{T}}$ when using the standard approach of arterial sampling for kinetic modelling [95•]. For PK, this is at least in part due to high binding to plasma proteins altered in proinflammatory states [98], and this may also be the case for second-generation ligands, albeit unconfirmed. The result is considerable variability in $V_{\mathrm{T}}$ within and across studies, and high vascular TSPO binding is further implicated to substantially affect outcome estimation [13•]. Limitations regarding the different generations of radioligands are summarised in Table 1.

The challenge of absolute quantification has lead researchers to find alternative methods to an arterial input function, including reference tissue modelling [95•]. However, unlike other ligands targeting neurotransmitter systems, there is not part of the brain parenchyma devoid of TSPO expression and so no true reference region exists [95•]. Nonetheless, different approaches have been sought. Considering PK, supervised clustering methods have enabled the identification of reference tissue [102]. While this is not applicable to second-generation ligands [81], a study using ${ }^{11}$ C-PBR28 in Alzheimer's patients was able to estimate binding using a cerebellar pseudo-reference region, demonstrating less variability compared with estimation using plasma activity [103]. Non-invasive techniques pose attractive alternatives to the less reliable arterial sampling in TSPO studies, but validation using such techniques is paramount concerning the ligand and population being studied.

\section{Future Directions}

Although the definitive functional role of TSPO continues to elude us, techniques such as X-ray crystallography have revealed its pentameric 3D structure, including selective bindings sites [104] [105]. These binding sites could prove novel therapeutic targets and models of CNS injury appear to show that PK has a neuroprotective effect with a reduction in reactive microglia and astrocytes [106, 107]. However, without steadfast knowledge on how the upregulation of TSPO influences the immune reaction, development of affective treatments will remain limited. For example, there is an evidence to suggest that TSPO may play a role as a negative regulator of inflammatory signalling in macrophages [108]. This supports the argument that increased TSPO binding, in certain circumstances, in fact, reflects a protective response rather than a pro-inflammatory, tissue-damaging phenotype, and microglial activation could be a normal response to independent pathological processes. This has significant therapeutic implications when considering TSPO-dependent agonistic versus antagonistic strategies, and exploration for alternative PET-based ligands (Table.2) targeting sensitive markers of glial activation $[1 \bullet \cdot$, as well as the immune response, will be crucial to progress in this field.

\section{Conclusion}

It is increasingly apparent that neuroinflammation is implicated in a diverse range of neurological and neuropsychiatric conditions. Despite the discussed limitations, it should be apparent that TSPO has emerged as an important neuroinflammatory biomarker for disease monitoring in vivo and contributing to our understanding of the manifestation chronic disease states.

While the development of novel techniques should be encouraged, neuroimaging researchers should continue to build on existing methods to drive innovation forward and overcome the inherent shortcomings in TSPO PET. Through the accurate depiction of the pathophysiological processes, we can not only improve diagnostic techniques, but develop new and effective immunomodulatory treatments, with the exciting potential of future neurorestorative therapies.

\section{Compliance with Ethical Standards}

Conflict of Interest Laura Best, Christine Ghadery, Nicola Pavese, Yen Foung Tai and Antonio P. Strafella each declare no potential conflicts of interest.

Human and Animal Rights and Informed Consent This article does not contain any studies with human or animal subjects performed by any of the authors.

Open Access This article is distributed under the terms of the Creative Commons Attribution 4.0 International License (http:// creativecommons.org/licenses/by/4.0/), which permits unrestricted use, distribution, and reproduction in any medium, provided you give appropriate credit to the original author(s) and the source, provide a link to the Creative Commons license, and indicate if changes were made.

\section{References}

Papers of particular interest, published recently, have been highlighted as:

- Of importance

•• Of major importance

1.• Tronel C, Largeau B, Santiago Ribeiro MJ, Guilloteau D, Dupont A-C, Arlicot N. Molecular targets for PET imaging of activated microglia: the current situation and future expectations. Int J Mol Sci. 2017;18(4). https://doi.org/10.2290/ijms18040802. The 
authors discuss the progress that has been made in the field of TSPO PET imaging, including the limitations of the current radioligands. They provide a detailed overview of alternative molecular targets, which may lead to future progress in the field of imaging neuroinflammation.

2.• Dupont A-C, Largeau B, Santiago Ribeiro MJ, Guilloteau D, Tronel C, Arlicot N. Translocator protein-18 $\mathrm{kDa}$ (TSPO) positron emission tomography (PET) imaging and its clinical impact in neurodegenerative diseases. Int J Mol Sci. 2017;18(4):785-822. The authors outline the in vivo PET TSPO imaging of neurological and neuropsychiatric conditions. This is a comprehensive summary of studies to date, including major advances and the impact it has had on our understanding of the pathology of these conditions.

3. Block ML, Zecca L, Hong J-S. Microglia-mediated neurotoxicity: uncovering the molecular mechanisms. Nat Rev Neurosci. 2007;8(1):57-69.

4. Hanisch U-K, Kettenmann H. Microglia: active sensor and versatile effector cells in the normal and pathologic brain. Nat Neurosci. 2007;10(11):1387-94.

5. Narayan N, Mandhair H, Smyth E, Dakin SG, Kiriakidis S, Wells $\mathrm{L}$, et al. The macrophage marker translocator protein (TSPO) is down-regulated on pro-inflammatory 'M1' human macrophages. PLoS One. 2017;12(10):e0185767.

6. Braestrup C, Squires RF. Specific benzodiazepine receptors in rat brain characterized by high-affinity $(3 \mathrm{H})$ diazepam binding. Proc Natl Acad Sci U S A. 1977;74(9):3805-9.

7. Banati RB. Visualising microglial activation in vivo. Glia. 2002;40(2):206-17.

8. Rojas S, Martín A, Arranz MJ, Pareto D, Purroy J, Verdaguer E, et al. Imaging brain inflammation with [ ${ }^{11}$ C]PK11195 by PET and induction of the peripheral-type benzodiazepine receptor after transient focal ischemia in rats. J Cereb Blood Flow Metab. 2007;27(12):1975-86.

9. Winkeler A, Boisgard R, Martin A, Tavitian B. Radioisotopic imaging of neuroinflammation. J Nucl Med. 2010;51(1):1-4.

10. Gatliff J, Campanella M. TSPO is a REDOX regulator of cell mitophagy. Biochem Soc Trans. 2015;43(4):543-52.

11. Veenman L, Shandalov Y, Gavish M. VDAC activation by the $18 \mathrm{kDa}$ translocator protein (TSPO), implications for apoptosis. J Bioenerg Biomembr. 2008;40(3):199-205.

12. Chauveau F, Boutin H, Van Camp N, Dollé F, Tavitian B. Nuclear imaging of neuroinflammation: a comprehensive review of [11C]PK11195 challengers. Eur J Nucl Med Mol Imaging. 2008;35(12):2304-19.

13. Turkheimer FE, Rizzo G, Bloomfield PS, Howes O, ZanottiFregonara P, Bertoldo A, et al. The methodology of TSPO imaging with positron emission tomography. Biochem Soc Trans. 2015;43(4):586-92. This review details the methodological aspects of TSPO PET imaging, including TSPO genetics and cellular heterogeneity, detailing how such factors impact quantification of TSPO PET data.

14.• Fan Z, Calsolaro V, Atkinson RA, Femminella GD, Waldman A, Buckley C, et al. Flutriciclamide (18F-GE180) PET: first-inhuman PET study of novel third-generation in vivo marker of human translocator protein. J Nucl Med Off Publ Soc Nucl Med. 2016;57(11):1753-9. The first published study investigating the kinetic properties of a third-generation TSPO ligand.

15. Ikawa M, Lohith TG, Shrestha S, Telu S, Zoghbi SS, Castellano S, et al. ${ }^{11} \mathrm{C}$-ER176, a radioligand for 18-kDa translocator protein, has adequate sensitivity to robustly image all three affinity genotypes in human brain. J Nucl Med. 2017;58(2):320-5.

16. Dulamea AO. Role of oligodendrocyte dysfunction in demyelination, remyelination and neurodegeneration in multiple sclerosis. Adv Exp Med Biol. 2017;958:91-127.
17. Gao Z, Tsirka SE. Animal models of MS reveal multiple roles of microglia in disease pathogenesis. Neurol Res Int. 2011;2011:1-9.

18. Politis M, Giannetti P, Su P, Turkheimer F, Keihaninejad S, Wu K, et al. Increased PK11195 PET binding in the cortex of patients with MS correlates with disability. Neurology. 2012;79(6):52330.

19. Herranz E, Giannì C, Louapre C, Treaba CA, Govindarajan ST, Ouellette R, et al. Neuroinflammatory component of gray matter pathology in multiple sclerosis. Ann Neurol. 2016;80(5):776-90. This study investigated ${ }^{11} \mathrm{C}$-PBR28 binding and its relationship to predicting poor clinical outcome and neurodegeneration in MS.

20. Debruyne JC, Versijpt J, Van Laere KJ, De Vos F, Keppens J, Strijckmans K, et al. PET visualization of microglia in multiple sclerosis patients using [11C]PK11195. Eur J Neurol. 2003;10(3): 257-64.

21. Colasanti A, Guo Q, Muhlert N, Giannetti P, Onega M, Newbould $\mathrm{RD}$, et al. In vivo assessment of brain white matter inflammation in multiple sclerosis with (18)F-PBR111 PET. J Nucl Med Off Publ Soc Nucl Med. 2014;55(7):1112-8.

22. Giannetti P, Politis M, Su P, Turkheimer F, Malik O, Keihaninejad $\mathrm{S}$, et al. Microglia activation in multiple sclerosis black holes predicts outcome in progressive patients: an in vivo $[(11) \mathrm{C}](\mathrm{R})$ PK11195-PET pilot study. Neurobiol Dis. 2014;65:203-10.

23. Oh U, Fujita M, Ikonomidou VN, Evangelou IE, Matsuura E, Harberts E, et al. Translocator protein PET imaging for glial activation in multiple sclerosis. J Neuroimmune Pharmacol Off J Soc NeuroImmune Pharmacol. 2011;6(3):354-61.

24. Vowinckel E, Reutens D, Becher B, Verge G, Evans A, Owens T, et al. PK11195 binding to the peripheral benzodiazepine receptor as a marker of microglia activation in multiple sclerosis and experimental autoimmune encephalomyelitis. J Neurosci Res. 1997;50(2):345-53.

25. Banati RB, Newcombe J, Gunn RN, Cagnin A, Turkheimer F, Heppner F, et al. The peripheral benzodiazepine binding site in the brain in multiple sclerosis: quantitative in vivo imaging of microglia as a measure of disease activity. Brain J Neurol. 2000;123(11):2321-37.

26. Versijpt J, Debruyne JC, Van Laere KJ, De Vos F, Keppens J, Strijckmans K, et al. Microglial imaging with positron emission tomography and atrophy measurements with magnetic resonance imaging in multiple sclerosis: a correlative study. Mult Scler Houndmills Basingstoke Engl. 2005;11(2):127-34.

27. Datta G, Violante IR, Scott G, Zimmerman K, Santos-Ribeiro A, Rabiner EA, et al. Translocator positron-emission tomography and magnetic resonance spectroscopic imaging of brain glial cell activation in multiple sclerosis. Mult Scler Houndmills Basingstoke Engl. 2017;23(11):1469-78.

28. Takano A, Piehl F, Hillert J, Varrone A, Nag S, Gulyás B, et al. In vivo TSPO imaging in patients with multiple sclerosis: a brain PET study with [18F]FEDAA1106. EJNMMI Res. 2013;3(1):30.

29. Park E, Gallezot J-D, Delgadillo A, Liu S, Planeta B, Lin S-F, et al. (11)C-PBR28 imaging in multiple sclerosis patients and healthy controls: test-retest reproducibility and focal visualization of active white matter areas. Eur J Nucl Med Mol Imaging. 2015;42(7): 1081-92.

30. Singhal T, O'Connor K, Dubey S, Belanger A, Hurwitz S, Chu R, et al. [F-18]PBR06 PET to assess TSPO binding and its association with brain atrophy and disability in multiple sclerosis. Neurology. 2018;90(15):P3.386.

31. Singhal T, Weiner HL, Bakshi R. TSPO-PET imaging to assess cerebral microglial activation in multiple sclerosis. Semin Neurol. 2017;37(5):546-57. Authors review the current status of TSPO PET imaging in MS and discuss the future direction for research on this field. 
32. Airas L, Dickens AM, Elo P, Marjamäki P, Johansson J, Eskola O, et al. In vivo PET imaging demonstrates diminished microglial activation after fingolimod treatment in an animal model of multiple sclerosis. J Nucl Med Off Publ Soc Nucl Med. 2015;56(2): 305-10.

33. Ratchford JN, Endres CJ, Hammoud DA, Pomper MG, Shiee N, McGready J, et al. Decreased microglial activation in MS patients treated with glatiramer acetate. J Neurol. 2012;259(6):1199-205.

34. Bonsack F, Alleyne CH, Sukumari-Ramesh S. Augmented expression of TSPO after intracerebral hemorrhage: a role in inflammation? J Neuroinflammation. 2016;13(1):151.

35. Iadecola C, Anrather J. The immunology of stroke: from mechanisms to translation. Nat Med. 2011;17(7):796-808.

36. Hu X, Li P, Guo Y, Wang H, Leak RK, Chen S, et al. Microglia/ macrophage polarization dynamics reveal novel mechanism of injury expansion after focal cerebral ischemia. Stroke. 2012;43(11):3063-70.

37. Thiel A, Radlinska BA, Paquette C, Sidel M, Soucy J-P, Schirrmacher R, et al. The temporal dynamics of poststroke neuroinflammation: a longitudinal diffusion tensor imaging-guided PET study with 11C-PK11195 in acute subcortical stroke. J Nucl Med Off Publ Soc Nucl Med. 2010;51(9):1404-12.

38. Schroeter M, Jander S, Witte OW, Stoll G. Heterogeneity of the microglial response in photochemically induced focal ischemia of the rat cerebral cortex. Neuroscience. 1999;89(4):1367-77.

39. Walberer M, Jantzen SU, Backes H, Rueger MA, Keuters MH, Neumaier B, et al. In-vivo detection of inflammation and neurodegeneration in the chronic phase after permanent embolic stroke in rats. Brain Res. 2014;1581:80-8.

40. Walter HL, Walberer M, Rueger MA, Backes H, Wiedermann D, Hoehn M, et al. In vivo analysis of neuroinflammation in the late chronic phase after experimental stroke. Neuroscience. 2015;292: 71-80.

41. Gulyás B, Tóth M, Schain M, Airaksinen A, Vas A, Kostulas K, et al. Evolution of microglial activation in ischaemic core and periinfarct regions after stroke: a PET study with the TSPO molecular imaging biomarker $[((11)) C]$ vinpocetine. J Neurol Sci. 2012;320(1-2):110-7.

42. Price CJS, Wang D, Menon DK, Guadagno JV, Cleij M, Fryer T, et al. Intrinsic activated microglia map to the peri-infarct zone in the subacute phase of ischemic stroke. Stroke. 2006;37(7):174953.

43. Pappata S, Levasseur M, Gunn RN, Myers R, Crouzel C, Syrota A, et al. Thalamic microglial activation in ischemic stroke detected in vivo by PET and [11C]PK1195. Neurology. 2000;55(7):10524.

44. Gerhard A, Schwarz J, Myers R, Wise R, Banati RB. Evolution of microglial activation in patients after ischemic stroke: a [11C](R)PK11195 PET study. NeuroImage. 2005;24(2):591-5.

45. Gulyas B, Toth M, Vas A, Shchukin E, Kostulas K, Hillert J, et al. Visualising neuroinflammation in post-stroke patients: a comparative PET study with the TSPO molecular imaging biomarkers [11C]PK11195 and [11C]vinpocetine. Curr Radiopharm. 2012;5(1):19-28

46. Ribeiro M-J, Vercouillie J, Debiais S, Cottier J-P, Bonnaud I, Camus V, et al. Could (18) F-DPA-714 PET imaging be interesting to use in the early post-stroke period? EJNMMI Res. 2014;4: 28.

47. Chaney A, Cropper HC, Johnson EM, Lechtenberg KJ, Peterson TC, Stevens MY, et al. 11C-DPA-713 versus 18F-GE-180: a preclinical comparison of translocator protein $18 \mathrm{kDa}$ PET tracers to visualize acute and chronic neuroinflammation in a mouse model of ischemic stroke. J Nucl Med Off Publ Soc Nucl Med. 2019;60(1):122-8. This study provides the first direct comparison of second-generation TSPO ligands in acute and chronic stroke using a murine model to determine the most sensitive and accurate for monitoring in vivo spatiotemporal dynamics of neuroinflammation in stroke.

48. Boutin H, Pinborg LH. TSPO imaging in stroke: from animal models to human subjects. Clin Transl Imaging. 2015 Dec;3(6): 423-35.

49. Donat CK, Scott G, Gentleman SM, Sastre M. Microglial activation in traumatic brain injury. Front Aging Neurosci. 2017;9:208. This article reviews the role of microglial activation in TBI in both human and animal models, describing the in vivo imaging of microglia and novel potential restorative therapeutic approaches in TBI.

50. Stemper BD, Pintar FA. Biomechanics of concussion. Prog Neurol Surg. 2014;28:14-27.

51. Blennow K, Hardy J, Zetterberg $\mathrm{H}$. The neuropathology and neurobiology of traumatic brain injury. Neuron. 2012;76(5):886-99.

52. Masel BE, DeWitt DS. Traumatic brain injury: a disease process, not an event. J Neurotrauma. 2010;27(8):1529-40.

53. Donat CK, Gaber K, Meixensberger J, Brust P, Pinborg LH, Hansen $\mathrm{HH}$, et al. Changes in binding of [(123)I]CLINDE, a high-affinity translocator protein $18 \mathrm{kDa}$ (TSPO) selective radioligand in a rat model of traumatic brain injury. NeuroMolecular Med. 2016;18(2):158-69.

54. Schwarzmaier SM, Plesnila N. Contributions of the immune system to the pathophysiology of traumatic brain injury - evidence by intravital microscopy. Front Cell Neurosci. 2014;8:358.

55. Roberts GW, Gentleman SM, Lynch A, Murray L, Landon M, Graham DI. Beta amyloid protein deposition in the brain after severe head injury: implications for the pathogenesis of Alzheimer's disease. J Neurol Neurosurg Psychiatry. 1994;57(4): 419-25.

56. Scott G, Hellyer PJ, Ramlackhansingh AF, Brooks DJ, Matthews PM, Sharp DJ. Thalamic inflammation after brain trauma is associated with thalamo-cortical white matter damage. J Neuroinflammation. 2015;12(1):224.

57. Jafari S, Etminan M, Aminzadeh F, Samii A. Head injury and risk of Parkinson disease: a systematic review and meta-analysis. Mov Disord Off J Mov Disord Soc. 2013;28(9):1222-9.

58. McMillan TM, Teasdale GM, Weir CJ, Stewart E. Death after head injury: the 13 year outcome of a case control study. J Neurol Neurosurg Psychiatry. 2011;82(8):931-5.

59. Giunta B, Obregon D, Velisetty R, Sanberg PR, Borlongan CV, Tan J. The immunology of traumatic brain injury: a prime target for Alzheimer's disease prevention. J Neuroinflammation. 2012;9: 185.

60. Maxwell WL, MacKinnon M-A, Stewart JE, Graham DI. Stereology of cerebral cortex after traumatic brain injury matched to the Glasgow outcome score. Brain J Neurol. 2010;133(1):139 60

61. Chiu C-C, Liao Y-E, Yang L-Y, Wang J-Y, Tweedie D, Karnati $\mathrm{HK}$, et al. Neuroinflammation in animal models of traumatic brain injury. J Neurosci Methods. 2016;272:38-49.

62. Ramlackhansingh AF, Brooks DJ, Greenwood RJ, Bose SK, Turkheimer FE, Kinnunen KM, et al. Inflammation after trauma: microglial activation and traumatic brain injury. Ann Neurol. 2011;70(3):374-83.

63. Johnson VE, Stewart JE, Begbie FD, Trojanowski JQ, Smith DH, Stewart W. Inflammation and white matter degeneration persist for years after a single traumatic brain injury. Brain $\mathrm{J}$ Neurol. 2013;136(1):28-42.

64. Folkersma H, Boellaard R, Yaqub M, Kloet RW, Windhorst AD, Lammertsma AA, et al. Widespread and prolonged increase in (R)-(11)C-PK11195 binding after traumatic brain injury. J Nucl Med Off Publ Soc Nucl Med. 2011;52(8):1235-9.

65. Coughlin JM, Wang Y, Munro CA, Ma S, Yue C, Chen S, et al. Neuroinflammation and brain atrophy in former NFL players: an 
in vivo multimodal imaging pilot study. Neurobiol Dis. 2015;74: $58-65$.

66. Coughlin JM, Wang Y, Minn I, Bienko N, Ambinder EB, Xu X, et al. Imaging of glial cell activation and white matter integrity in brains of active and recently retired National Football League Players. JAMA Neurol. 2017;74(1):67-74. This study demonstrates that localised brain injury and repair, indicated by increased TSPO binding and white matter changes, is present in young NFL players with incidence of concussion.

67. Israel I, Ohsiek A, Al-Momani E, Albert-Weissenberger C, Stetter C, Mencl S, et al. Combined [18F]DPA-714 micro-positron emission tomography and autoradiography imaging of microglia activation after closed head injury in mice. J Neuroinflammation. 2016;13(1):140. The study demonstrates that ${ }^{\mathbf{1 8}}$ F-DPA-714 uptake in PET imaging correlated with trauma severity, metabolic deficits and brain microglial activation following closed head TBI.

68. Hu X, Leak RK, Shi Y, Suenaga J, Gao Y, Zheng P, et al. Microglial and macrophage polarization - new prospects for brain repair. Nat Rev Neurol. 2015;11(1):56-64.

69. Wang G, Shi Y, Jiang X, Leak RK, Hu X, Wu Y, et al. HDAC inhibition prevents white matter injury by modulating microglia/ macrophage polarization through the GSK3 $\beta /$ PTEN/Akt axis. Proc Natl Acad Sci U S A. 2015;112(9):2853-8.

70. Olmos-Alonso A, Schetters STT, Sri S, Askew K, Mancuso R, Vargas-Caballero M, et al. Pharmacological targeting of CSF1R inhibits microglial proliferation and prevents the progression of Alzheimer's-like pathology. Brain J Neurol. 2016;139(3):891907.

71. Venneti S, Wagner AK, Wang G, Slagel SL, Chen X, Lopresti BJ, et al. The high affinity peripheral benzodiazepine receptor ligand DAA1106 binds specifically to microglia in a rat model of traumatic brain injury: implications for PET imaging. Exp Neurol. 2007;207(1):118-27.

72. Hammoud DA, Endres CJ, Chander AR, Guilarte TR, Wong DF, Sacktor NC, et al. Imaging glial cell activation with [11C]-RPK11195 in patients with AIDS. J Neuro-Oncol. 2005 Aug;11(4):346-55.

73. Vera JH, Guo Q, Cole JH, Boasso A, Greathead L, Kelleher P, et al. Neuroinflammation in treated HIV-positive individuals: A TSPO PET study. Neurology. 2016;12;86(15):1425-32. This study demonstrates that increased levels of brain inflammation correlate with poorer cognitive outcomes despite effective treatment.

74. Wiley CA, Lopresti BJ, Becker JT, Boada F, Lopez OL, Mellors J, et al. Positron emission tomography imaging of peripheral benzodiazepine receptor binding in human immunodeficiency virusinfected subjects with and without cognitive impairment. J Neuro-Oncol. 2006;12(4):262-71.

75. Garvey LJ, Pavese N, Politis M, Ramlackhansingh A, Brooks DJ, Taylor-Robinson SD, et al. Increased microglia activation in neurologically asymptomatic HIV-infected patients receiving effective ART. AIDS Lond Engl. 2014;28(1):67-72.

76. Monji A, Kato T, Kanba S. Cytokines and schizophrenia: microglia hypothesis of schizophrenia. Psychiatry Clin Neurosci. 2009;63(3):257-65.

77. van Berckel BN, Bossong MG, Boellaard R, Kloet R, Schuitemaker A, Caspers E, et al. Microglia activation in recentonset schizophrenia: a quantitative (R)-[11C]PK11195 positron emission tomography study. Biol Psychiatry. 2008;64(9):820-2.

78. Doorduin J, de Vries EFJ, Willemsen ATM, de Groot JC, Dierckx RA, Klein HC. Neuroinflammation in schizophrenia-related psychosis: a PET study. J Nucl Med Off Publ Soc Nucl Med. 2009;50(11):1801-7.

79. Kenk M, Selvanathan T, Rao N, Suridjan I, Rusjan P, Remington $\mathrm{G}$, et al. Imaging neuroinflammation in gray and white matter in schizophrenia: an in-vivo PET study with [18F]-FEPPA. Schizophr Bull. 2015;41(1):85-93.

80. Takano A, Arakawa R, Ito H, Tateno A, Takahashi H, Matsumoto $\mathrm{R}$, et al. Peripheral benzodiazepine receptors in patients with chronic schizophrenia: a PET study with [11C]DAA1106. Int J Neuropsychopharmacol. 2010;13(7):943-50.

81. Bloomfield PS, Selvaraj S, Veronese M, Rizzo G, Bertoldo A, Owen DR, et al. Microglial activity in people at ultra high risk of psychosis and in schizophrenia: an [(11)C]PBR28 PET brain imaging study. Am J Psychiatry. 2016;173(1):44-52.

82. Coughlin JM, Wang Y, Ambinder EB, Ward RE, Minn I, Vranesic $\mathrm{M}$, et al. In vivo markers of inflammatory response in recent-onset schizophrenia: a combined study using [(11)C]DPA-713 PET and analysis of CSF and plasma. Transl Psychiatry. 2016;6:e777.

83. Hafizi S, Tseng H-H, Rao N, Selvanathan T, Kenk M, Bazinet RP, et al. Imaging microglial activation in untreated first-episode psychosis: a PET study with [18F]FEPPA. Am J Psychiatry. 2017;174(2):118-24.

84. Selvaraj S, Bloomfield PS, Cao B, Veronese M, Turkheimer F, Howes OD. Brain TSPO imaging and gray matter volume in schizophrenia patients and in people at ultra high risk of psychosis: an [11C]PBR28 study. Schizophr Res. 2018;195:206-14. This study demonstrates a negative correlation between TSPO signal and total grey matter volume, suggesting that microglial activity is related to altered cortical volume observed in schizophrenia.

85. Hannestad J, DellaGioia N, Gallezot J-D, Lim K, Nabulsi N, Esterlis I, et al. The neuroinflammation marker translocator protein is not elevated in individuals with mild-to-moderate depression: a $\left[{ }^{11}\right.$ C]PBR28 PET study. Brain Behav Immun. 2013;33: $131-8$.

86. Setiawan E, Wilson AA, Mizrahi R, Rusjan PM, Miler L, Rajkowska G, et al. Role of translocator protein density, a marker of neuroinflammation, in the brain during major depressive episodes. JAMA Psychiatry. 2015;72(3):268-75.

87. Richards EM, Zanotti-Fregonara P, Fujita M, Newman L, Farmer $\mathrm{C}$, Ballard ED, et al. PET radioligand binding to translocator protein (TSPO) is increased in unmedicated depressed subjects. EJNMMI Res. 2018;8(1):57. This recently published study identified a trend towards increased TSPO binding in patients with MDE, which is significant in non-medicated, but not in medicated, MDE patients.

88. Holmes SE, Hinz R, Conen S, Gregory CJ, Matthews JC, AntonRodriguez JM, et al. Elevated translocator protein in anterior cingulate in major depression and a role for inflammation in suicidal thinking: a positron emission tomography study. Biol Psychiatry. 2018;83(1):61-9.

89.• Setiawan E, Attwells S, Wilson AA, Mizrahi R, Rusjan PM, Miler $\mathrm{L}$, et al. Association of translocator protein total distribution volume with duration of untreated major depressive disorder: a crosssectional study. Lancet Psychiatry. 2018;5(4):339-47. This study demonstrates that microglial activation, as identified using TSPO PET, is greater in patients with chronologically advanced MDE who have had longer periods with no antidepressant treatment, suggesting a different illness phase. It also highlights that when anti-depressant treatment is commenced, the observed yearly increase in microglial activation is no longer observed.

90. Horikawa H, Kato TA, Mizoguchi Y, Monji A, Seki Y, Ohkuri T, et al. Inhibitory effects of SSRIs on IFN- $\gamma$ induced microglial activation through the regulation of intracellular calcium. Prog Neuro-Psychopharmacol Biol Psychiatry. 2010;34(7):1306-16.

91. Tynan RJ, Weidenhofer J, Hinwood M, Cairns MJ, Day TA, Walker FR. A comparative examination of the anti-inflammatory effects of SSRI and SNRI antidepressants on LPS stimulated microglia. Brain Behav Immun. 2012;26(3):469-79. 
92. Li H, Sagar AP, Kéri S. Translocator protein (18 kDa TSPO) binding, a marker of microglia, is reduced in major depression during cognitive-behavioral therapy. Prog NeuroPsychopharmacol Biol Psychiatry. 2018;83:1-7.

93. Haarman BCMB, Riemersma-Van der Lek RF, de Groot JC, Ruhé HGE, Klein HC, Zandstra TE, et al. Neuroinflammation in bipolar disorder - a [(11)C]-(R)-PK11195 positron emission tomography study. Brain Behav Immun. 2014;40:219-25.

94. Haarman BCM, Burger H, Doorduin J, Renken RJ, SibeijnKuiper AJ, J-BC M, et al. Volume, metabolites and neuroinflammation of the hippocampus in bipolar disorder - a combined magnetic resonance imaging and positron emission tomography study. Brain Behav Immun. 2016;56:21-33.

95. Albrecht DS, Granziera C, Hooker JM, Loggia ML. In vivo imaging of human neuroinflammation. ACS Chem Neurosci. 2016;7(4):470-83. This review summarises the current practices in neuroimaging for investigating CNS neuroinflammation beyond TSPO PET.

96. Chen M-K, Guilarte TR. Imaging the peripheral benzodiazepine receptor response in central nervous system demyelination and remyelination. Toxicol Sci Off J Soc Toxicol. 2006;91(2):532-9.

97. Cosenza-Nashat M, Zhao M-L, Suh H-S, Morgan J, Natividad R, Morgello S, et al. Expression of the translocator protein of $18 \mathrm{kDa}$ by microglia, macrophages and astrocytes based on immunohistochemical localization in abnormal human brain. Neuropathol Appl Neurobiol. 2009;35(3):306-28.

98. Lockhart A, Davis B, Matthews JC, Rahmoune H, Hong G, Gee A, et al. The peripheral benzodiazepine receptor ligand PK11195 binds with high affinity to the acute phase reactant alpha1-acid glycoprotein: implications for the use of the ligand as a CNS inflammatory marker. Nucl Med Biol. 2003;30(2):199-206.

99. Le Fur G, Perrier ML, Vaucher N, Imbault F, Flamier A, Benavides J, et al. Peripheral benzodiazepine binding sites: effect of PK 11195, 1-(2-chlorophenyl)-N-methyl-N-(1-methylpropyl)3 -isoquinolinecarboxamide. I In vitro studies. Life Sci. 1983;32(16):1839-47.

100. Fujimura Y, Zoghbi SS, Simeon FG, Taku A, Pike VW, Innis RB, et al. Quantification of translocator protein $(18 \mathrm{kDa})$ in the human brain with PET and a novel radioligand, 18F-PBR06. J Nucl Med. 2009;50(7):1047-53.
101. Fujimura Y, Ikoma Y, Yasuno F, Suhara T, Ota M, Matsumoto R, et al. Quantitative analyses of 18F-FEDAA1106 binding to peripheral benzodiazepine receptors in living human brain. J Nucl Med Off Publ Soc Nucl Med. 2006;47(1):43-50.

102. Turkheimer FE, Edison P, Pavese N, Roncaroli F, Anderson AN, Hammers A, et al. Reference and target region modeling of [11C](R)-PK11195 brain studies. J Nucl Med Off Publ Soc Nucl Med. 2007;48(1):158-67.

103. Lyoo CH, Ikawa M, Liow J-S, Zoghbi SS, Morse CL, Pike VW, et al. Cerebellum can serve as a pseudo-reference region in Alzheimer disease to detect neuroinflammation measured with PET Radioligand binding to translocator protein. J Nucl Med Off Publ Soc Nucl Med. 2015;56(5):701-6.

104. Korkhov VM, Sachse C, Short JM, Tate CG. Three-dimensional structure of TspO by electron cryomicroscopy of helical crystals. Structure. 2010;18(6):677-87.

105. Li F, Liu J, Zheng Y, Garavito RM, Ferguson-Miller S. Crystal structures of translocator protein (TSPO) and mutant mimic of a human polymorphism. Science. 2015;347(6221):555-8.

106. Veiga S, Azcoitia I, Garcia-Segura LM. Ro5-4864, a peripheral benzodiazepine receptor ligand, reduces reactive gliosis and protects hippocampal hilar neurons from kainic acid excitotoxicity. J Neurosci Res. 2005;80(1):129-37.

107. Ryu JK, Choi HB, McLarnon JG. Peripheral benzodiazepine receptor ligand PK11195 reduces microglial activation and neuronal death in quinolinic acid-injected rat striatum. Neurobiol Dis. 2005;20(2):550-61.

108. Wang W, Zhang L, Zhang X, Xue R, Li L, Zhao W, et al. Lentiviral-mediated overexpression of the $18 \mathrm{kDa}$ translocator protein (TSPO) in the hippocampal dentate gyrus ameliorates LPS-induced cognitive impairment in mice. Front Pharmacol. 2016;7:384.

Publisher's Note Springer Nature remains neutral with regard to jurisdictional claims in published maps and institutional affiliations. 